\title{
KLOE results on light meson properties
}

\section{B. Di Micco*}

I.N.F.N. sezione di Roma Tre

E-mail: dimiccodfis.uniroma3.it

\section{for the KLOE collaboration ${ }^{\dagger}$}

\begin{abstract}
We present KLOE results on hadronic physics. We show results on the $\phi \rightarrow a_{0}(980) \gamma$ decay, with $a_{0} \rightarrow \eta \pi^{0}$, which provides the $a_{0}(980)$ couplings to $\eta \pi$ and $K^{+} K^{-}$and we discuss their relation with the $a_{0}$ structure; we also show the final measurement for the search of the $\phi \rightarrow K^{0} \bar{K}^{0} \gamma$ decay, which proceeds through $f_{0}(980) / a_{0}(980) \gamma$. Results for the upper limit on the branching ratio are presented with the full KLOE statistics, which allow to test most of the available theoretical models. Here we update our measurement of the gluonium content of the $\eta^{\prime}$ that indicated a $3 \sigma$ 's evidence. Other SU(3) relations were added in the fit, further allowing the extraction of other parameters, such as the vector mixing angle and the SU(3) breaking parameter. Finally we show final results for the BR of the $\eta \rightarrow \pi^{+} \pi^{-} e^{+} e^{-}$decay, for the CP violating asymmetry in the angular distribution between the $\pi^{+} \pi^{-}$and the $e^{+} e^{-}$decay planes in the $\eta$ rest frame, whose observation could test unexpected mechanism of CP violation, thus providing an hint of new physics beyond the Standard Model. The upper limit on the strength of the new interaction is also evaluated.
\end{abstract}

The 2009 Europhysics Conference on High Energy Physics,

July 16 - 222009

Krakow, Poland

\footnotetext{
${ }^{*}$ Speaker.

${ }^{\dagger}$ F. Ambrosino, A. Antonelli, M. Antonelli, F. Archilli, P. Beltrame, G. Bencivenni, C. Bini, C. Bloise, S. Bocchetta, F. Bossi, P. Branchini, G. Capon, T. Capussela, F. Ceradini, P. Ciambrone, E. De Lucia, A. De Santis, P. De Simone, G. De Zorzi, A. Denig, A. Di Domenico, C. Di Donato, B. Di Micco, M. Dreucci, G. Felici, S. Fiore, P. Franzini, C. Gatti, P. Gauzzi, S. Giovannella, E. Graziani, M. Jacewicz, G. Lanfranchi, J. Lee-Franzini, M. Martini, P. Massarotti, S. Meola, S. Miscetti, M. Moulson, S. Müller, F. Murtas, M. Napolitano, F. Nguyen, M. Palutan, A. Passeri, V. Patera, P. Santangelo, B. Sciascia, T. Spadaro, L. Tortora, P. Valente, G. Venanzoni, R.Versaci, G. Xu.
} 


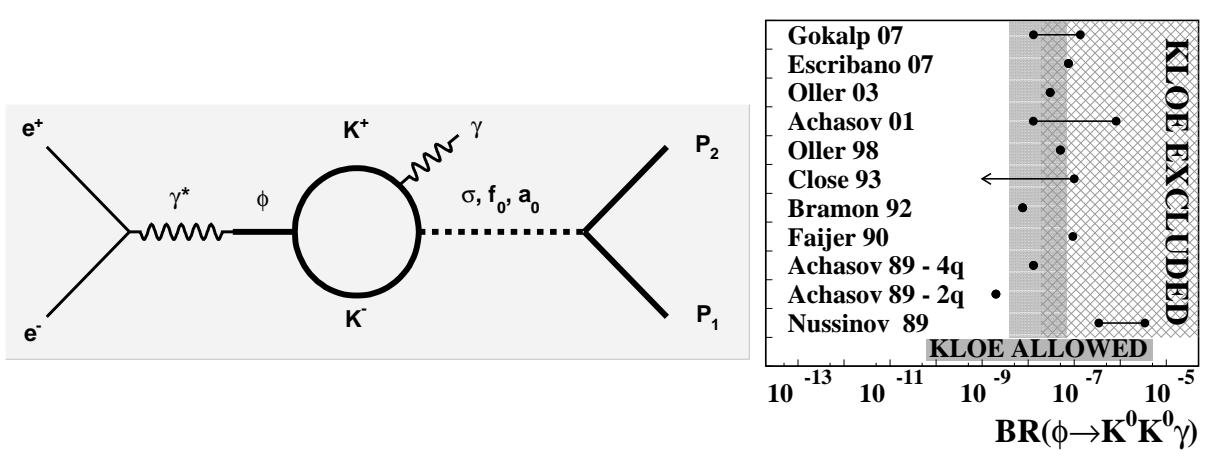

Figure 1: left Feynman diagram of the $\phi$ production and decay to the scalar mesons according to the Kaon loop model; right $f_{0} \rightarrow \bar{K}^{0} K^{0}$ upper limit compared with the theoretical predictions (see [8] for references), the KLOE allowed region has been computed using the $a_{0}, f_{0}$ parameters measured by KLOE.

\section{Introduction}

KLOE is a general pourpose detector working at the Frascati $\phi$ factori DAФNE. DAФNE is an $e^{+} e^{-}$collider working at $\sqrt{s}=1020 \mathrm{GeV}$ corresponding to the $\phi$ meson mass. In the whole KLOE data taking an integrated luminosity of $2.5 \mathrm{fb}^{-1}$ was collected. It corresponds to 8 billions of $\phi$ meson produced and 100 millions of $\eta$ mesons. The KLOE apparatus consist of two main subdetectors. A calorimeter [1] with a cylindrical shape with $2 \mathrm{~m}$ radius and $4 \mathrm{~m}$ height, whose axis is parallel to the beam direction. The calorimeter energy and time resoultions are:

$$
\frac{\sigma_{E}}{E}=\frac{5.7 \%}{\sqrt{E(\mathrm{GeV})}} \quad \sigma_{t}=\frac{54 \mathrm{ps}}{\sqrt{E(\mathrm{GeV})}} \oplus 140 \mathrm{ps}
$$

The second subdetector is a stereo wires drift chamber [2] which allow 3D reconstruction of the tracks. The gas mixture is $90 \% \mathrm{He}$ and $10 \% \mathrm{iC}_{4} \mathrm{H}_{4}$. The drift chamber is immersed in a magnetic field of $0.5 \mathrm{~T}$. The $p_{t}$ resolution is $0.5 \%$ while the vertex resoultion along the beam pipe is $2 \mathrm{~mm}$.

\section{The $a_{0}$ and the nature of the scalar mesons.}

Scalar mesons can be interpreted as $q \bar{q}$ pair or 4 quarks states[3]. Here we describe the measurement of the couplings of the $a_{0}$ to $\eta \pi^{0}$ that together with our previous measurments of the decays $\phi \rightarrow S \gamma \rightarrow \pi^{0} \pi^{0} \gamma, \pi^{+} \pi^{-} \gamma$ [4, 5] can be used to identify the nature of the scalars.

The coupling of the scalars to the pseudoscalars can be extracted from the decay $\phi \rightarrow S \gamma \rightarrow$ $P_{1} P_{2} \gamma\left(P_{1}\right.$ and $P_{2}$ being pseudoscalar mesons) using the Kaon loop model (fig. 1, left). The decay $\phi \rightarrow \eta \pi^{0} \gamma$ is identified through two different decays of the $\eta$ meson: $\eta \rightarrow \gamma \gamma$ and $\eta \rightarrow \pi^{+} \pi^{-} \pi^{0}$. Fitting the $M_{\eta \pi^{0}}$ invariant mass distribution we have determined the couplings of the $a_{0}$ to $K^{+} K^{-}$ and $\eta \pi^{0}$. The couplings of the scalars to the pseudoscalars can be used to determine the structure of the scalar mesons in the naive $\mathrm{SU}(3)$ hypothesis. $\mathrm{SU}(3)$ predicts the couplings with different quark structure assumption, but both $4 q$ and 2 quark model are not able to reproduce the ratio between the couplings of $f_{0}$ and $a_{0}$ to $K^{+} K^{-}$[6]. Recently a new model for scalars has been developed [7]. In this model two amplitudes participate to the scalar decay, the interference between them keeps 


\begin{tabular}{|c|c|c|c|}
\hline & Measured & $q q \bar{q} \bar{q}$ & $q \bar{q}$ \\
\hline$g f_{0 K^{+} K^{-}}(\mathrm{GeV})$ & $4.0-4.7^{1}$ & $c_{i}=-2.8--3.4 \mathrm{GeV}^{-1}$ & $c_{i}=-4.0-4.8 \mathrm{GeV}^{-1}$ \\
\hline$g f_{0 \pi^{+} \pi^{-}}(\mathrm{GeV})$ & $-1.8-2.2^{1}$ & $c_{f}=20-24 \mathrm{GeV}^{-1}$ & $c_{f}=16-20 \mathrm{GeV}^{-1}$ \\
\hline$g a_{0 K^{+} K^{-}}(\mathrm{GeV})$ & $2.0-2.2^{1}$ & $2.1-2.5$ & $2.4-2.9$ \\
\hline$g a_{0 \eta \pi}(\mathrm{GeV})$ & $2.5-2.8^{1}$ & $3.3-3.9$ & $6.6-7.9$ \\
\hline
\end{tabular}

Table 1: Instanton model prediction of the scalar couplings to the pseudoscalars. The measurements ${ }^{1}$ are from KLOE.

the value of $\mathrm{ga}_{0 K^{+} K^{-}}$low. In table 1 we have computed the predictions of the instanton model for the $a_{0}$ couplings using KLOE data for $f_{0}, a_{0}$ mass and the $f_{0}$ couplings to the pseudoscalars. The values obtained are compared to the $a_{0}$ couplings measured, showing a good agreement in the $4 q$ hypothesis.

\section{Search for $\phi \rightarrow\left(f_{0}+a_{0}\right) \gamma \rightarrow K^{0} \bar{K}^{0} \gamma$ decay.}

Both $a_{0}$ and $f_{0}$ couple to the $K^{+} K^{-}$and $K^{0} \bar{K}^{0}$ pseudoscalars, so that the decay $\phi \rightarrow\left(f_{0}+\right.$ $\left.a_{0}\right) \gamma \rightarrow K^{0} \bar{K}^{0} \gamma$ is possible. SU(3) relates the coupling constant to the psudoscalars in a simple way: $g f_{0 \pi^{+} \pi^{-}}=2 g f_{0 \pi^{0} \pi^{0}}, g f_{0 K^{0} \bar{K}^{0}}=g f_{0 K^{+} K^{-}}, g a_{0 K^{0} \bar{K}^{0}}=-g a_{0 K^{+} K^{-}}$. Due to the opposite sign in the $g f_{0 K^{0} \bar{K}^{0}} \cdot g f_{0 K^{+} K^{-}}$and $g a_{0 K^{0} \bar{K}^{0}} \cdot g a_{0 K^{+} K^{-}}$, destructive interference is expected between $f_{0}$ and $a_{0}$. The scalars can decay both in $K_{s} K_{s}$ and $K_{L} K_{L}$ meson pairs. The state with two $K_{s}$ in the final state is identified in the $K_{s} \rightarrow \pi^{+} \pi^{-}$channel, looking for 4 tracks pointing to the Interaction Point. The main background comes from $\phi \rightarrow K_{s} K_{L}$ events where a CP violating decay $K_{L} \rightarrow \pi^{+} \pi^{-}$near the interaction point happens. KLOE [8] has analysed a sample of $2.2 \mathrm{fb}^{-1}$ of data observing 5 events with $3.2 \pm 0.7$ of expected background, obtaining $\operatorname{Br}\left(\phi \rightarrow\left(f_{0}+a_{0}\right) \gamma\right)<1.9 \times 10^{-8}$. The KLOE upper limit is shown in fig. 1, right.

\section{4. $\eta-\eta^{\prime}$ mixing and gluonium.}

The $\eta^{\prime}$ meson is considered a good candidate to host a gluon condensate. The $\eta$ and $\eta^{\prime}$ wave functions can be decomposed in three terms: the $u, d$ quark wave function $|N\rangle=1 / \sqrt{2}(|u \bar{u}\rangle+|d \bar{d}\rangle)$ the $S=|s \bar{s}\rangle$ component and the gluonium $|G\rangle$. The wave functions are written as:

$$
\left|\eta^{\prime}\right\rangle=\cos \psi_{G} \sin \psi_{P}|N\rangle+\cos \psi_{G} \cos \psi_{P}|S\rangle+\sin \psi_{G}|G\rangle \quad|\eta\rangle=\cos \psi_{P}|N\rangle-\sin \psi_{P}|S\rangle
$$

where $\psi_{P}$ is the $\eta-\eta^{\prime}$ mixing angle and $Z_{G}^{2}=\sin ^{2} \psi_{G}$ is the gluonium fraction in the $\eta^{\prime}$ meson. The $Z_{G}^{2}$ parameter can be interpreted as the mixing with a pseudoscalar glue ball. Recently [9] it has been identified with the $\eta(1405)$.

The gluonium content has been extracted fitting the widths of the magnetic dipole transition $V \rightarrow$ $P \gamma$, where $V$ are the vector mesons $\rho, \omega, \phi$ and $P$ the pseudoscalar mesons $\pi^{0}, \eta, \eta^{\prime}$, together with the $\pi^{0} \rightarrow \gamma \gamma$ and $\eta^{\prime} \rightarrow \gamma \gamma$ partial widths. In particular the KLOE measurement of $R_{\phi}=\operatorname{Br}(\phi \rightarrow$ $\left.\eta^{\prime} \gamma\right) / \operatorname{Br}(\phi \rightarrow \eta \gamma)$ has been used. All relevant formulae are shown in [10]. We report the final result in fig. 2 showing that the gluonium component $Z_{G}^{2}$ is at $3 \sigma$ from zero. 


\begin{tabular}{c|c}
\hline$\chi^{2} /$ n.d.f(Prob) & $4.6 / 3(20 \%)$ \\
$Z_{G}^{2}$ & $0.115 \pm 0.036$ \\
$\varphi_{P}$ & $(40.4 \pm 0.6)^{\circ}$ \\
$Z_{N S}$ & $0.936 \pm 0.025$ \\
$Z_{S}$ & $0.83 \pm 0.05$ \\
$\varphi_{V}$ & $(3.32 \pm 0.09)^{\circ}$ \\
$m_{s} / \bar{m}$ & $1.24 \pm 0.07$ \\
\hline
\end{tabular}

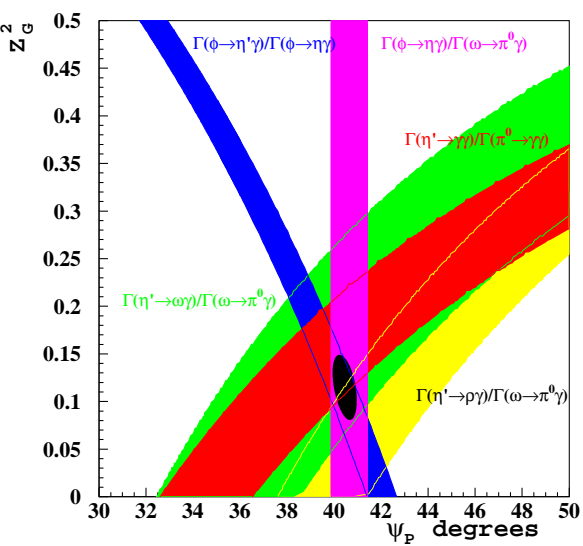

Figure 2: left fit results, right $68 \%$ C.L. region in the $\left(\psi_{P}, Z_{G}^{2}\right)$ plane.

\section{The $\eta \rightarrow \pi^{+} \pi^{-} e^{+} e^{-}$decay.}

In the standard model the $\eta \rightarrow \pi^{+} \pi^{-} e^{+} e^{-}$decay proceeds through an internal convertion of a photon in the $\eta$ meson field. It's branching ratio can be computed from the $\eta \rightarrow \pi^{+} \pi^{-} \gamma$ branching ratio and the $\eta$ form factor. Several low energy effective models have been used to compute it [11]. We have measured this branching ratio looking for 4 tracks in the drift chamber and one photon in the calorimeter. We have performed the most accurate branching ratio measurement with the result: $\operatorname{Br}\left(\eta \rightarrow \pi^{+} \pi^{-} e^{+} e^{-}\right)=\left(2.68 \pm 0.09_{\text {stat. }} \pm 0.07_{\text {syst. }}\right) \times 10^{-4}$. Furthermore this decay is a good candidate to reveal unconventional $\mathrm{CP}$ violation. In fact, $\mathrm{CP}$ violation produces an asymmetry in the distribution of the angle between the $\pi^{+} \pi^{-}$and $e^{+} e^{-}$ planes in the $\eta$ reference frame. Such an asymmetry can be produced by a 4 fermions operator [12] and is not constrained by other CP sensitive measurements. In particular we measure: $A_{\phi}=\left(-0.6 \pm 2.5_{\text {stat. }} \pm 1.8_{\text {syst. }}\right) \times 10^{-2}$ that puts a limit on the effective coupling of this operator $|G|<1.9$ at $90 \%$ C.L.

\section{References}

[1] M. Adinolfi, et al., Nucl. Instr. Meth. A482 (364) 2002;

[2] M. Adinolfi, et al., Nucl. Instr. Math A488 (51) 2002;

[3] R. L. Jaffe, Phys. Rev. D 15 (1977) 267;

[4] F. Ambrosino, et al. Phys. Lett. B634 (2006) 148;

[5] F. Ambrosino, et al. Eur. Phys. J. C49 (2007) 473;

[6] The KLOE collaboration, B. Di Micco, et al. Nucl.Phys.Proc.Suppl. 181-182 (2008) 215;

[7] G. 't Hooft, et al. Phys. Lett. B662 (2008) 424;

[8] F. Ambrosino, et al. Phys. Lett. B679 (2009) 10;

[9] H.-Y. Cheng, H.-n. Li and K.-F. Liu, Phys. Rev. D79 (2009) 014024;

[10] F. Ambrosino, et al. JHEP 0907 (2009) 105;

[11] F. Ambrosino, et al. Phys, Lett. B675 (2009)283;

[12] D.N. Gao, Mod. Phys. Lett. A 17 (2002) 1583 